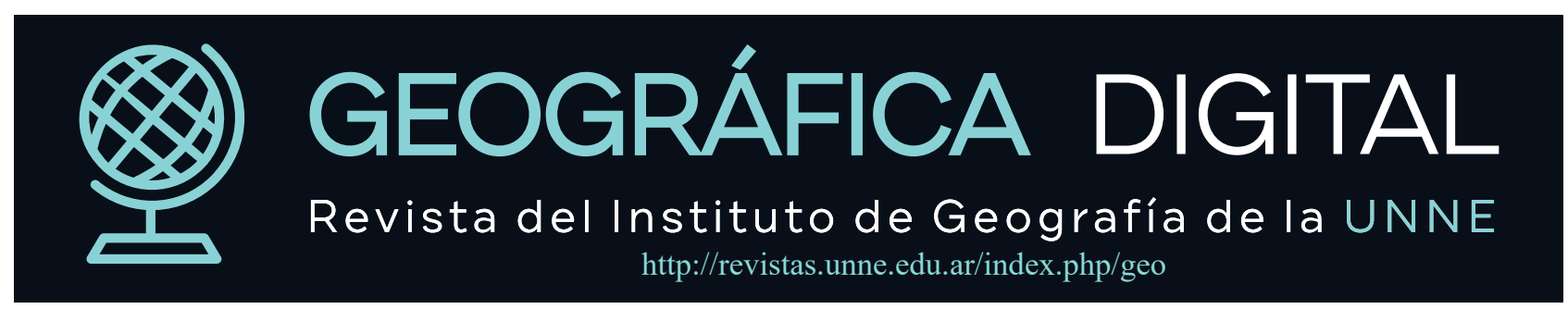

Revista Geográfica Digital, 2021, Vol. 18, No 35, 2-14 pp., E-ISSN: 1668-5180: DOI: http://dx.doi.org/10.30972/geo.18355130

\title{
Análisis espacial cuantitativo de los Condicionantes Socioespaciales de la Salud (CSS) de la megaciudad Buenos Aires
}

\section{Quantitative spatial analysis of Socio-spatial conditioning factors of health (SCfH) of the megacity Buenos Aires}

\section{Buzai, Gustavo}

Universidad Nacional de Luján, Instituto de Investigaciones Geográficas / Consejo Nacional de Investigaciones Científicas y Técnicas, CONICET. Argentina. gdb@unlu.edu.ar

Palabras claves: Geografía de la Salud Análisis espacial Clasificación espacial Megaciudad Buenos Aires SIG

\section{R E S U M E N}

El análisis espacial cuantitativo de los Condicionantes Socioespaciales de la Salud (CSS) es una aproximación teórico-metodológica que permite definir, desde un punto de vista geográfico, la distribución espacial de la vulnerabilidad social a enfermedades. Mediante la aplicación de Sistemas de Información Geográfica (SIG) es posible utilizar metodologías de análisis multivariado que llevan a la obtención del resultado final en un mapa síntesis. En la Argentina, la base de datos del Censo Nacional de Población, Hogares y Viviendas permite determinar un conjunto básico de variables que definen dimensiones de análisis centrales: Educación, Economía, Pobreza, Construcción, Servicios y Equipamiento. La combinación de estas dimensiones permite realizar el mapa que presenta la distribución espacial de los CSS de la Megaciudad Buenos Aires y definir su área de mayor criticidad, presentando estos resultados como base fundamental de la planificación territorial con la posibilidad de ser usado para la generación de acciones tendientes a la disminución de disparidades socioespaciales.

\section{A B S T R A C T}

Keywords:

Geography of Health

Spatial Analysis

Spatial Classification

Megacity Buenos Aires GIS
The quantitative spatial analysis of the Socio-spatial Conditioning factors of Health ( $\mathrm{SCfH}$ ) is a theoretical-methodological approach that allows to define, from a geographical perspective, the spatial distribution of social vulnerability to diseases. Through the application of Geographic Information Systems (GIS), is possible to use methods of multivariate analysis that lead to obtaining the final result in a map synthesis. In Argentina, the database of the National Census of Population, Households and Housing allows to determine a basic set of variables that define dimensions of central analysis: Education, Economy, Poverty, Construction, Services and Equipment. The combination of these dimensions generate the map presenting the spatial distribution of the CSS of the Megacity Buenos Aires and the most critical area, showing its spatial inequality as a fundamental basis of the territorial planning with the intention of generating actions for the reduction of disparities. 


\section{Introducción}

La inequidad en salud es una temática de gran relevancia actual en el estudio de la diferenciación socio-espacial urbana como apoyo a la planificación y gestión territorial. Tiene como sustento subyacente el 'mapa social urbano' en tanto aproximación general que permite avanzar en la definición de los condicionantes socio-espaciales de la salud (CSS) tendientes a la delimitación de áreas con diferencial vulnerabilidad poblacional a las enfermedades.

La Geografía, mediante el desarrollo de técnicas del análisis espacial cuantitativo aplicado con Sistemas de Información Geográfica (SIG), posibilita la obtención de resultados centrados en procesos de construcción regional. Considerando esta línea de análisis, el objetivo del presente trabajo es realizar el análisis espacial de los CSS y delimitar las áreas de mayor vulnerabilidad sanitaria en la Megaciudad Buenos Aires, Argentina.

Para realizarlo se encara una revisión de los aspectos teóricos centrales en cuanto a la discusión del papel de la dimensión espacial a partir de su núcleo conceptual relacionado al campo temático de la Geografía de la Salud y la definición de los CSS para un abordaje empírico.

La extensa superficie y magnitud poblacional posibilitan que las megaciudades presenten grandes disparidades internas, en este sentido, las metodologías de análisis multivariado permiten su construcción. La metodología de clasificación utilizada incluye un trabajo matricial que inicia con la tabla de atributos en datos originales obtenidos del censo nacional, su trasformación para obtener la comparabilidad y su síntesis a través de la obtención de un puntaje de clasificación para las unidades espaciales. Un posterior cálculo de autocorrelación espacial, amplía el resultado al discriminar el área con mayor vulnerabilidad.

En síntesis, llegar a la construcción de los CSS a partir de la combinación de atributos, permite contar con una herramienta de gran apoyo a una gestión diferencial que minimice la vulnerabilidad sociosanitaria de la población en el área más poblada y densa de la Argentina.

\section{Aspectos teóricos}

\subsection{Espacio geográfico y análisis espacial cuantitativo}

La Geografía actual cuenta con una gran valorización contextual ya que las diferentes ciencias sociales consideran que la dimensión espacial representada por el espacio geográfico constituye un primordial elemento explicativo de la realidad. La Geografía es la ciencia que tiene como objeto formal la perspectiva espacial y, a partir de ello, desarrolló gran cantidad de conceptos y metodologías para su abordaje.

Consideramos la aproximación espacial en las tres instancias conceptualizados por Piaget (2007) en cuanto a la evolución mental humana: 'espacio vivido' que se experimenta y descubre empíricamente, 'espacio percibido' que comienza a pensarse y 'espacio concebido' que se representa simbólicamente. Primero nos trasladamos, luego pensamos en distancias, orientaciones y finalmente se conceptualizan para ser dibujadas en un papel.

El ser humano se diferencia de toda la biología planetaria a través de su racionalidad en tanto animal simbólico y, en este sentido, el desarrollo de software y particularmente los SIG representan el punto culmine en el camino de la posibilidad de abordaje del espacio geográfico.

Desde un punto de vista cuantitativo y focalizado a partir del espacio geográfico concebido hacia el estudio del espacio vivido, pueden abordarse desde dos aproximaciones empíricas, según se lo considere en su característica de 'absoluto' como contenedor de objetos sobre la superficie terrestre o 'relativo' a partir de la interacción espacial horizontal producida entre ellos, puntos de inicio-destino de flujos que incluyen tiempos.

Estas dos perspectivas permiten contemplar los diferentes conceptos de abordaje de toda temática socioespacial. Los conceptos operativos del análisis espacial (Buzai, 2010; Buzai y Baxendale, 2011) son los de 'localización' (ubicación específica de cada objeto en un lugar -sitio- o en relación a otros objetos -posición-), 'distribución' (como se reparten los objetos sobre la superficie terrestre), 'asociación espacial' (grado de relación entre diferentes distribuciones), interacción espacial (análisis de flujos entre objetos), 'evolución espacial' (dimensión espacio-temporal) y ‘síntesis espacial'(combinación de las manifestaciones empíricas de los cinco conceptos anteriores). 
Los contextos espaciales junto a los conceptos centrales del análisis espacial son los que permiten dar respuesta a la vulnerabilidad socio-sanitaria de la población. La construcción modelística de la realidad se realiza a partir de un gran nivel de abstracción que obtiene concreción al momento de ser utilizado como herramienta en el ámbito de la planificación y gestión.

En síntesis, el análisis espacial cuantitativo es el paradigma científico que provee la perspectiva a partir de la cual abordamos la realidad y los SIG la principal tecnología en el estudio de la dimensión espacial de la Salud.

\subsection{Geografía y condicionantes socioespaciales de la salud}

El desarrollo histórico del estudio de la salud humana desde una perspectiva espacial produce como resultado un recorrido de más de dos siglos de extensión que fue presentado en diferentes instancias llegando hasta la construcción del campo temático de la Geografía de la Salud (Barcellos y Buzai, 2006; Buzai, 2015; Villerías Alarcón, 2017; Barcellos, Buzai y Santana, 2018).

Si bien la relación entre la salud y el ambiente se encontró presente de forma constante desde un punto de vista determinista desde los inicios de la Medicina a través de los estudios de Hipócrates de Cos (460 a.C. - 370 a.C.), esta relación, a partir de la primera revolución industrial (segunda mitad del siglo XVIII) se generaliza como la relación entre la población y su entorno, orientándose hacia la consideración del medio altamente antropizado de las ciudades.

Es de esta manera que el análisis de la salud humana comienza a realizarse a través de las condiciones de vida urbana teniendo focalización principal en la situación laboral y habitacional de las personas como aproximación a las condiciones de precariedad. En el ámbito urbano surgen relaciones causales entre la pobreza, la morbilidad y la mortalidad como base del inicio de la Epidemiología Social a través de las contribuciones de Louis René Villermé (Francia 1830), Edwin Chadwick e Friedrich Engels (Inglaterra 1840) y Rudolf Virchow (Alemania 1850).

Un estudio importante realizado en el marco de la transición desde el paradigma higienista al bacteriológico lo constituye la investigación realizada por John Snow (1813-1858), en la que son utilizadas las técnicas del modelado cartográfico por superposición temática para apoyar la hipótesis de que el cólera se transmite a través del agua. El mapa es presentado en Snow (1855) y constituye un trabajo pionero de resolución geográfica.

A inicios del siglo XX se destacan los aportes geográficos pioneros de Max Sorre y Jean Brunhes que con una mirada centrada en el ambiente realizaron sus estudios temáticos en el campo de la salud a partir de la distribución espacial de los complejos patógenos, un concepto sistémico que corresponde a la trama de las relaciones duraderas y estables entre los seres vivos, el hombre y su medio. El resultado final es el surgimiento de una enfermedad en determinados sitios de encuentro entre los agentes causales, vectores y huéspedes.

A pesar de que la filosofía dominante corresponde a la 'geografía regional' de carácter descriptivo, estas ideas avanzan con la posibilidad de realizar estudios desde un punto de vista sistémico y en ellos, el método de superposición cartográfica, según Sorre (1952), permite combinar todos los elementos naturales y antrópicos en la conformación del 'habitat'.

En 1949, el Congreso Internacional de Geografía realizado en Lisboa(Portugal) brinda reconocimiento oficial a la Geografía Médica, la cual se encontró apoyada en una reciente definición de ‘salud' de la OMS, la cual era considerada como un estado completo de bienestar físico, mental y social, y no únicamente como falta de enfermedad.

A través del aporte teórico de Jacques May se realiza una ampliación al concepto de complejos patógenos a través de los 'complejos geógenos', el cual incluye los aspectos socio-demográficos y económicos (May, 1950). El objetivo sigue siendo determinar las áreas de enfermedad y cartografiar su distribución espacial, con privilegio en el mundo subdesarrollado de clima tropical. En este sentido la Geografía de la Salud consistía en el estudio sistémico de las enfermedades de la tierra y las enfermedades de la población.

Durante la segunda mitad del siglo XX se produce la 'revolución cuantitativa' en Geografía. La existencia de una perspectiva racionalista basada en procedimientos de construcción regional cualitativa es considerada como primer escalón conceptual que luego se amplía con la introducción de métodos cuantitativos y la búsqueda de modelos y leyes científicas que brindan las pautas de distribución espacial. Encontrar las regularidades espaciales resultaba ser fundamental para avanzar en las tareas de planificación 
y gestión (Buzai y Baxendale, 2013).

En 1976 durante el congreso de la Unión Geográfica Internacional (IGU) realizado en Moscú se reconoce una reorientación y alcances de la especialidad. Surge la Geografía de la Salud incluyendo dos líneas de análisis bien definidas: el estudio de las enfermedades (Geografía Médica) y el de los centros de atención (Geografía de los Servicios Sanitarios). En la línea de esta perspectiva se produjo bibliografía general (Howe, 1985; Olivera, 1993; Pickenhayn, 2009; Anthamattem y Hazen, 2011; Cromley y McLafferty, 2011; Santana, 2014; Santana Juárez y Galindo Mendoza, 2014; Buzai, 2015; Gatrell y Elliott, 2015; Kanaroglu et al., 2015; Emch et al., 2017), desarrollos centrados en el análisis espacial de la distribución espacial de casos a través de la cartografía (Curto, 2003; Koch, 2014; Iñiguez y Barcellos, 2014), hacia los vínculos espaciales entre la oferta y demanda de servicios de atención (Garrocho, 1995; Lang, 2002; Ramírez, 2005) y sobre el papel de las actuales tecnologías digitales aplicada en el análisis espacial de la salud (Ramírez, 2004; Buzai, 2009; Seguinot Barbosa, 2014).

En el año de 1986 se lleva a cabo la primera conferencia internacional sobre la Promoción de la Salud en Ottawa, donde se emite una carta para todos los países y se establecen las condiciones y requisitos como la paz, la educación, la vivienda, la alimentación, la renta, un ecosistema estable, la justicia social y la equidad para establecer condiciones de salud óptimas. Que de acuerdo con la OMS (2010) los factores políticos, económicos, sociales, culturales, el medio ambiente, los estilos de vida y biológicos intervienen en favor o detrimento de la salud.

La Organización Mundial de la Salud (OMS) en 2005 formó una comisión que se encargó de estudiar más a fondo los factores sociales y ambientales que incidían en la salud para así elaborar recomendaciones y mejorarla, cabe resaltar que se le denomino Comisión de Determinantes Sociales de la Salud. Los DSS son las circunstancias en que las personas nacen, crecen, viven, trabajan y envejecen, donde éste contexto es resultado de la distribución del dinero, el poder y los recursos dentro de las tres escalas (mundial, nacional y local) para actuar sobre las disparidades en salud (OMS, 2010; Castro, 2011) y se convirtieron centrales en el análisis espacial de la salud (Álvarez Castaño, 2009; Santos Padrón, 2011; Fuenzalida et al., 2013; Cordero y Murayama, 2013; Mejía, 2015; Villerías Alarcón, 2017).

Por lo que, la comisión planteó un marco conceptual de las principales categorías y vías de acción de los determinantes, que enmarca el contexto socio-político en un nivel macro (determinantes estructurales), la posición social y económica, y condiciones materiales ambas en el nivel individual (determinantes intermedios), que en conjunto generan alteraciones en la salud y en el bienestar humano. Es así que con ayuda de los CSS se muestra un panorama de los problemas de salud en la sociedad, esto debido a la distribución desigual del poder, los ingresos, los bienes y los servicios, que afectan a la salud y que expresan la desigualdad dentro de la misma sociedad.

En un análisis orientado a la crítica de la perspectiva extrema a la que alude la teoría del 'determinismo', Buzai y Santana Juárez (2018) no consideran que la salud se encuentre determinada en una relación permanente y unívoca, sino que debe pensarse en una situación de un determinismo atenuado (parcial) a través de los Condicionantes Socioespaciales de la Salud (CSS). Consideramos que este concepto presenta una propuesta de mayor amplitud y realismo. Modera la perspectiva determinista y la lleva hacia un determinismo parcial ubicado entre el determinismo y el indeterminismo, más cercano al primero que al segundo, e incorpora la dimensión espacial con lo cual amplía el contexto de la salud pudiendo abarcar teóricamente la totalidad de potenciales condicionantes contextuales.

Más allá de la biología y del estilo de vida, todo condicionamiento contextual será socioespacial en un tiempo específico, condiciones que influyen en la situación de salud, pero no la determinan, ya que siempre existe un margen de incertidumbre que surge de las características individuales y de las respuestas sociales ante los problemas de la salud.

\section{Definiciones operativas}

\section{1. Área de estudio}

La Megaciudad Buenos Aires es la mayor área urbana de la Argentina. Ubicada sobre el Río de la Plata presenta un frente costero de aproximadamente $200 \mathrm{~km}$. de extensión y un radio teórico que genera un semicírculo con promedio de $60 \mathrm{~km}$. desde el centro principal (CBD, Central Bussines District), denominado la 'city porteña'.

La mayoría de las investigaciones que se realizaron sobre este espacio lo hicieron generalmente 
en el continuo urbano definido como Gran Buenos Aires (GBA) (INDEC, 2003), el cual incluye a la Ciudad Autónoma de Buenos Aire como ciudad central (Capital Federal) y 24 municipios de la Provincia de Buenos Aires que la rodean y que contienen el área urbana transjurisdiccional.

Unos años después, la Dirección Provincial de Ordenamiento Urbano y Territorial (DPOUyT, 2007) amplió oficialmente su definición espacial al considerar el espacio funcional de los movimientos poblacionales cotidianos en el área y así contempla incorporar 16 municipios anexos al GBA tradicional. En Buzai y Montes Galbán (2020), a partir del uso de imágenes satelitales nocturnas y como avance respecto de la aplicación realizada por Montes Galbán (2020), se define el último proceso de expansión y conurbación mediante el cual el continuo urbano puede establecerse en cinco ejes principales donde el de mayor extensión llega a la distancia aproximada de $90 \mathrm{~km}$.

Considerando la conformación del mosaico municipal, la Megaciudad Buenos Aires se encuentra compuesta por 41 unidades espaciales (UE) (Figura 1) que tienen una superficie de $14.188 \mathrm{~km} 2 \mathrm{con}$ 14.839.026 habitantes:

Tabla 1. Unidades espaciales de la Megaciudad Buenos Aires.

\begin{tabular}{|c|c|l|}
\hline UE & Entidad geográfica & \multicolumn{1}{c|}{ Municipios } \\
\hline 1 & Capital Federal & Ciudad Autónoma de Buenos Aires (CABA) \\
\hline 24 & $\begin{array}{c}\text { Municipios del GBA } \\
\text { tradicional }\end{array}$ & $\begin{array}{l}\text { Almirante Brown, Avellaneda, Berazategui, Esteban Echeverría, Ezeiza, } \\
\text { Florencio Varela, General San Martín, Hurlingham, Ituzaingó, José C. Paz, } \\
\text { La Matanza, Lanús, Lomas de Zamora, Malvinas Argentinas, Merlo, Moreno, } \\
\text { Morón, Quilmes, San Fernando, San Isidro, San Miguel, Tigre, Tres de Febrero } \\
\text { y Vicente López }\end{array}$ \\
\hline 16 & $\begin{array}{c}\text { Municipios conurbados al } \\
\text { GBA tradicional }\end{array}$ & $\begin{array}{l}\text { Berisso, Brandsen, Campana, Cañuelas, Ensenada, Escobar, Excaltación de } \\
\text { la Cruz, General Las Heras, General Rodríguez, La Plata, Luján, Marcos Paz, } \\
\text { Pilar, Presidente Perón, San Vicente y Zárate }\end{array}$ \\
\hline
\end{tabular}

Fuente: elaboración propia.

Figura 1. Área de estudio.

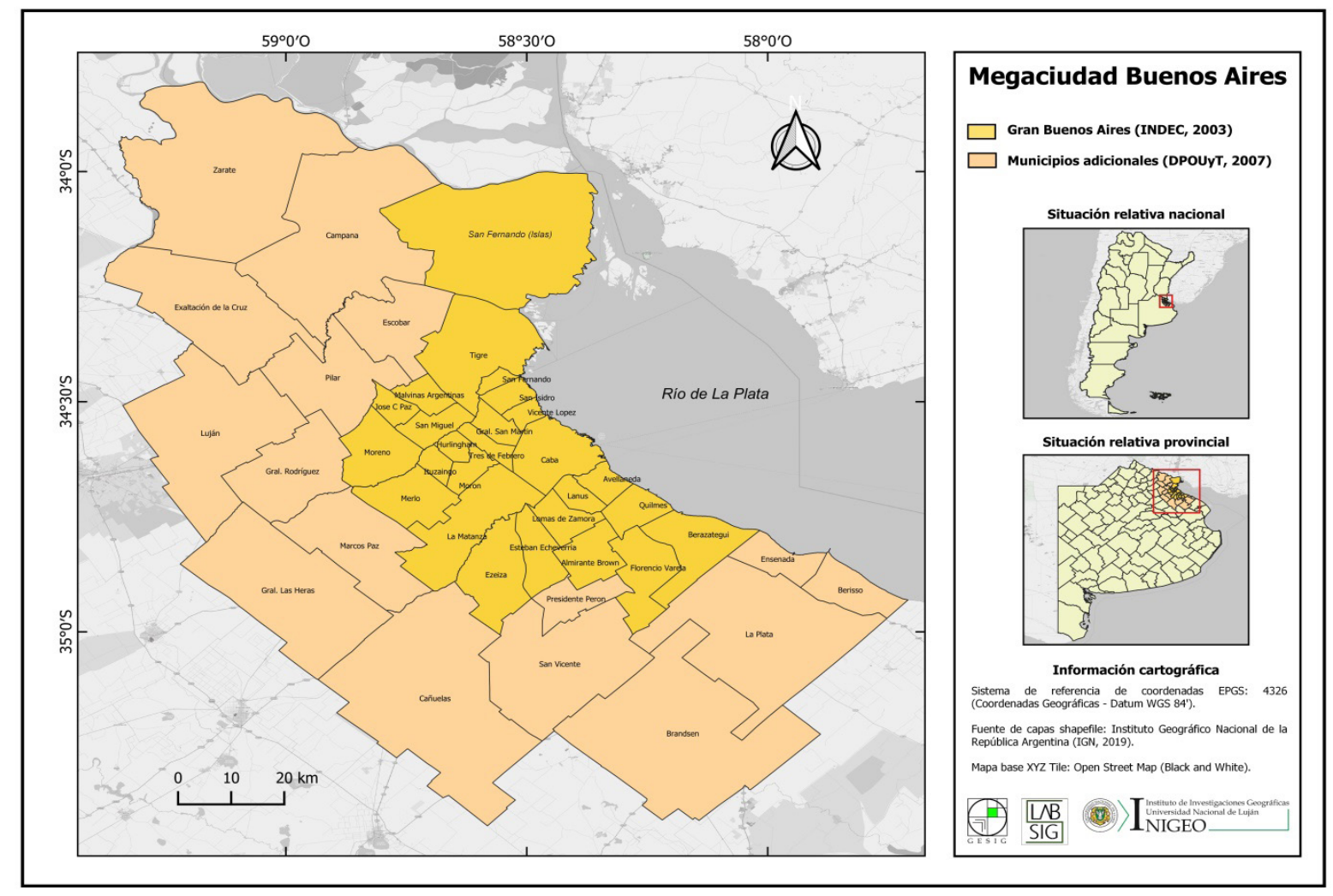

Fuente: cartografía de Eloy Montes Galbán (INIGEO-CONICET) 


\subsection{Variables}

Las enfermedades generalmente se relacionan con los agentes que la provocan aunque, en realidad, existen múltiples factores que contribuyen a que esta situación tenga diferencial intensidad, desde un punto de vista espacial aquellos relacionados con cuestiones socio-demográficas y de habitabilidad. Corresponde a poblaciones que deben enfrentar situaciones adversas a partir de bases contextuales diferentes.

El enfoque de los CSS intenta verificar estos contextos a través de sus principales dimensiones, entre ellas Educación, Economía, Pobreza, Construcción, Servicios y Equipamiento, y desde la Geografía son consideradas en base a la distribución espacial resultante. Incidir en la estructura resultante permitirá mitigar los problemas de la salud de la población y que en última instancia estas disparidades espaciales son inequidades propias del mapa social.

Las variables que permiten definir cada dimensión son consideradas de costo, es decir, que en todos los casos, sus máximos puntajes indican las situaciones más desfavorables.

La Tabla 2 presenta el sistema de variables utilizados a partir de las posibilidades de selección que brinda la base de datos REDATAM del Censos Nacional de Población, Hogares y Viviendas 2010 de la República Argentina (INDEC, 2013).

Tabla 2. Variables para el estudio de los CSS.

\begin{tabular}{|c|c|c|c|}
\hline Contexto & Dimensiones & Variables & Categorías \\
\hline \multirow{5}{*}{ SOCIO-DEMOGRÁFICO } & \multirow[b]{2}{*}{ Educación } & Sabe leer y escribir - No & \multirow{6}{*}{$\begin{array}{c}\text { Muy alto } \\
\text { Alto } \\
\text { Medio }\end{array}$} \\
\hline & & $\begin{array}{c}\text { Condición de asistencia escolar - Nunca } \\
\text { asistió }\end{array}$ & \\
\hline & Economía & Condición de actividad - Desocupados & \\
\hline & \multirow[b]{2}{*}{ Pobreza } & Hogares con NBI & \\
\hline & & $\begin{array}{l}\text { Hacinamiento crítico - más de } 3 \text { personas } \\
\text { por cuarto }\end{array}$ & \\
\hline \multirow{5}{*}{ HABITABILIDAD } & \multirow[b]{2}{*}{ Construcción } & Población en rancho o casilla & \\
\hline & & $\begin{array}{l}\text { Material predominante de los pisos: tierra, } \\
\text { ladrillo suelto }\end{array}$ & Bajo \\
\hline & \multirow[b]{2}{*}{ Servicios } & Tenencia de agua - fuera del terreno & \multirow[t]{3}{*}{ Muy bajo } \\
\hline & & $\begin{array}{c}\text { Desagüe del inodoro - a hoyo, excavación en } \\
\text { la tierra }\end{array}$ & \\
\hline & Equipamiento & Equipamiento - heladera: No. & \\
\hline
\end{tabular}

Fuente: elaboración propia.

Los datos básicos de cada variable para las 1.305 fracciones censales de los municipios que contienen la megaciudad Buenos Aires, en valores absolutos, se presenta en la Tabla 3.

Tabla 3. Estadísticas básicas en fracciones censales.

\begin{tabular}{|l|c|c|c|c|}
\hline \multicolumn{1}{|c|}{ Variables } & Suma & Mínimo & Máximo & Media \\
\hline Población que no lee ni escribe & 814.175 & 0 & 2.737 & 623,89 \\
\hline Población que nunca asistió (educación) & 299.899 & 0 & 1.472 & 229,81 \\
\hline Población desocupada & 465.357 & 0 & 1.273 & 356,59 \\
\hline Población con NBI & 1.703 .297 & 0 & 9.473 & $1.305,21$ \\
\hline Hogares con hacinamiento crítico & 161.776 & 0 & 939 & 123,97 \\
\hline Hogares con rancho o casilla & 577.905 & 0 & 6.131 & 442,84 \\
\hline Hogares con piso de tierra & 36.224 & 0 & 1.082 & 27,76 \\
\hline Hogares con agua fuera del terreno & 54.594 & 0 & 742 & 41,83 \\
\hline Hogares con desagüe hoyo & 12.296 & 0 & 209 & 9,42 \\
\hline Hogares sin heladera & 165.658 & 0 & 1.806 & 126,94 \\
\hline
\end{tabular}

Fuente: elaboración propia. 
Tabla 4. Valores extremos de dimensiones (en porcentajes).

\begin{tabular}{|l|c|c|c|}
\hline & Mínimo & Máximo & Media \\
\hline 1. Educación & 0 & 95,64 & 19,20 \\
\hline 2. Economía & 0 & 100 & 23,02 \\
\hline 3. Pobreza & 0 & 94,67 & 13,49 \\
\hline 4. Construcción & 0 & 85,58 & 4,90 \\
\hline 5. Servicios & 0 & 84,94 & 5,07 \\
\hline 6. Equipamiento & 0 & 100 & 7,03 \\
\hline
\end{tabular}

Fuente: elaboración propia.

\section{Aspectos metodológicos}

\subsection{Correlación vertical: matrices y síntesis}

La base de datos en su nivel cartográfico está formada por 1.305 polígonos que corresponden a las fracciones censales de los municipios de la Megaciudad Buenos Aires y en su nivel alfanumérico por gran cantidad de variables, donde 10 fueron seleccionadas para el estudio.

La operatividad de la base de datos para llevar a cabo esta investigación constituye de 4 etapas (Buzai, 2014). La primera corresponde a la creación de una matriz de los datos originales (MDO) a partir de los datos censales, la cual va a estar conformada por las unidades espaciales, que dentro de un SIG será representada por unidades espaciales en forma de filas por 10 variables en las columnas.

Se crea la matriz de datos índice (MDI) llevado los valores a porcentajes como peso relativo del valor de la variable en la unidad espacial, según el origen del dato, relacionado al total de población u hogares. De esta forma se logra un primer nivel de comparabilidad. Posteriormente se crea la matriz de puntaje estándar (MDE) a partir del cálculo del puntaje omega, en la cual se transforman todos los datos de cada variable (ver Ecuación 1), llevándolos a una misma unidad de medida y con ellos hacerlos perfectamente comparables:

$$
\Omega=\frac{X-m}{M-m} * 100
$$

Ecuación 1. Puntaje Omega.

Donde $X$ es el dato de cada unidad espacial, $m$ y $M$ son respectivamente los datos menor y mayor de la serie de datos. El procedimiento transforma los datos al rango de 0 a 100, siendo la MDE la base para la obtención del PCE con la síntesis espacial de la problemática en estudio.

Una vez determinadas las dimensiones a partir del cálculo del promedio de las variables que las componen se aplica el cálculo para la obtención de puntaje de clasificación espacial (PCE) como síntesis a partir del cálculo del promedio de las dimensiones estandarizadas (Dim $\Omega)$ :

$$
P C E=\frac{\sum \operatorname{Dim} \Omega}{n}
$$

Ecuación 2. Puntaje de Clasificación Espacial.

El procedimiento brinda como resultados parciales 6 mapas correspondientes a cada una de las dimensiones y un mapa síntesis, también estandarizado, de vulnerabilidad a partir de las dimensiones de los CSS.

\subsection{Correlación horizontal: autocorrelación espacial}

El concepto de autocorrelación espacial se sustenta en la afirmación que considera que, en el espacio geográfico, todo se encuentra relacionado con todo, pero los elementos más cercanos están más 
relacionados entre sí que con los más lejanos ('Ley de Tobler'). La metodología ha sido detallada como uno de los procedimientos centrales del análisis espacial por diferentes autores (Gámir Orueta, Ruiz Pérez y Seguí Pons, 1995; Celemín, 2009; Buzai y Baxendale, 2012).

El análisis de autocorrelación espacial corresponde a una aplicación univariada de vecindad a partir de considerar cada unidad espacial como central respecto de las que tengan con ella relación de contigüidad.

El principal índice utilizado para medir la autocorrelación espacial es el I de Moran (Ecuación 3), actualmente satisfactoriamente incorporado en SIG (Anselin, 2003). Su propósito es comparar los valores de cada localización con los valores presentados en las unidades espaciales contiguas:

$$
I=\frac{n \sum_{i}\left(x_{i}-\bar{x}\right) \sum_{j} w_{j}\left(x_{j}-\bar{x}\right)}{\left(\sum_{i} \sum_{j} w_{j}\right) \sum_{i}\left(x_{i}-\bar{x}\right)^{2}}
$$

Ecuación 3. I de Moran.

Donde $n$ es el número de unidades espaciales, $x_{i}$ es el valor de la variable en la unidad espacial $i, x_{i}$ es el valor de la variable en la otra localización, $\bar{x}$ es la media de la variable, $\mathrm{y} w_{i}$ es una ponderación que indica la relación de contigüidad entre las unidades espaciales $i, j$. Si ambas son limítrofes $w_{i j}=1$, si no $w_{i j}$ $=0$.

Es posible profundizar el análisis a partir de la identificación de patrones locales de asociación espacial a partir del cálculo LISA (Local Indicators of Spatial Association) (Anselin, 1995) como método que permite descomponer el índice y verificar cuanto contribuye cada unidad espacial a la información del valor global. LISA (L) en la localización espacial para la variable es una función de los valores observado en sus unidades espaciales limítrofes .

$$
L_{i}=f\left(x_{i}, x_{j}\right)
$$

Ecuación 4. LISA.

Los límites para cada observación están tomados de la matriz de contigüidad espacial. El avance en la metodología permite determinar cúmulos espaciales locales denominados hot-spots y cold-spots en agrupamientos de valores altos y bajos respectivamente de acuerdo a la aplicación:

$$
I_{i}=z_{i} \sum w_{j} z_{j}
$$

Ecuación 5. Matriz de Contigüidad Espacial.

Las mediciones se realizan a partir de observaciones en datos estandarizados $z_{i}$ y $z_{j}$ para unidades espaciales contiguas definidas por los pesos de $w_{i j^{\prime}}$ De esta manera valores positivos y negativos estarían indicando relaciones espaciales similares y contrapuestas respectivamente.

\section{Resultados y análisis}

\subsection{Distribución espacial de los CSS}

La distribución espacial evidenciada a través de la cartografía temática, es una herramienta de gran importancia en apoyo al direccionamiento geográfico de políticas públicas que tengan como objetivo la disminución de disparidades socioespaciales.

Los principales conceptos utilizados en esta aplicación son los de distribución espacial mediante mapas temáticos, asociación espacial en el cálculo de correlación, síntesis espacial mediante el PCE e interacción espacial con la autocorrelación espacial. El proceso lleva a conocer la diferenciación espacial interna de la Megaciudad Buenos Aires y estructura de la vulnerabilidad de la población en cuanto a los CSS. 
La distribución espacial de las dimensiones se presenta en la serie cartográfica presentada en la Figura 2. Son mapas realizados mediante el método de clasificación por cortes naturales en cinco categorías de tipo ordenada con diferente intensidad de colores en correspondencia a la intensidad del dato representado.

Figura 2. Distribución espacial de las dimensiones

(n)

Fuente: elaboración propia.

Mientras la vulnerabilidad en las dimensiones Educación, Construcción, Servicios y Equipamientos tienen una tendencia hacia la dispersión rural, las dimensiones Economía y pobreza muestran una predominancia urbana, en este último caso con contigüidad al Gran Buenos Aires. Se destaca con mayor desfavorabilidad un semicírculo intermedio de la megaciudad que apoya con valores altos sobre el frente costero en ambos extremos.

\subsection{Distribución espacial sintética}

La Figura 3 presenta la distribución espacial sintética a partir de la combinación de las 6 dimensiones utilizadas en el cálculo del PCE de los CSS y la Figura 4 despeja los resultados a partir de las relaciones de vecindad en las semejanzas entre las unidades espaciales. 


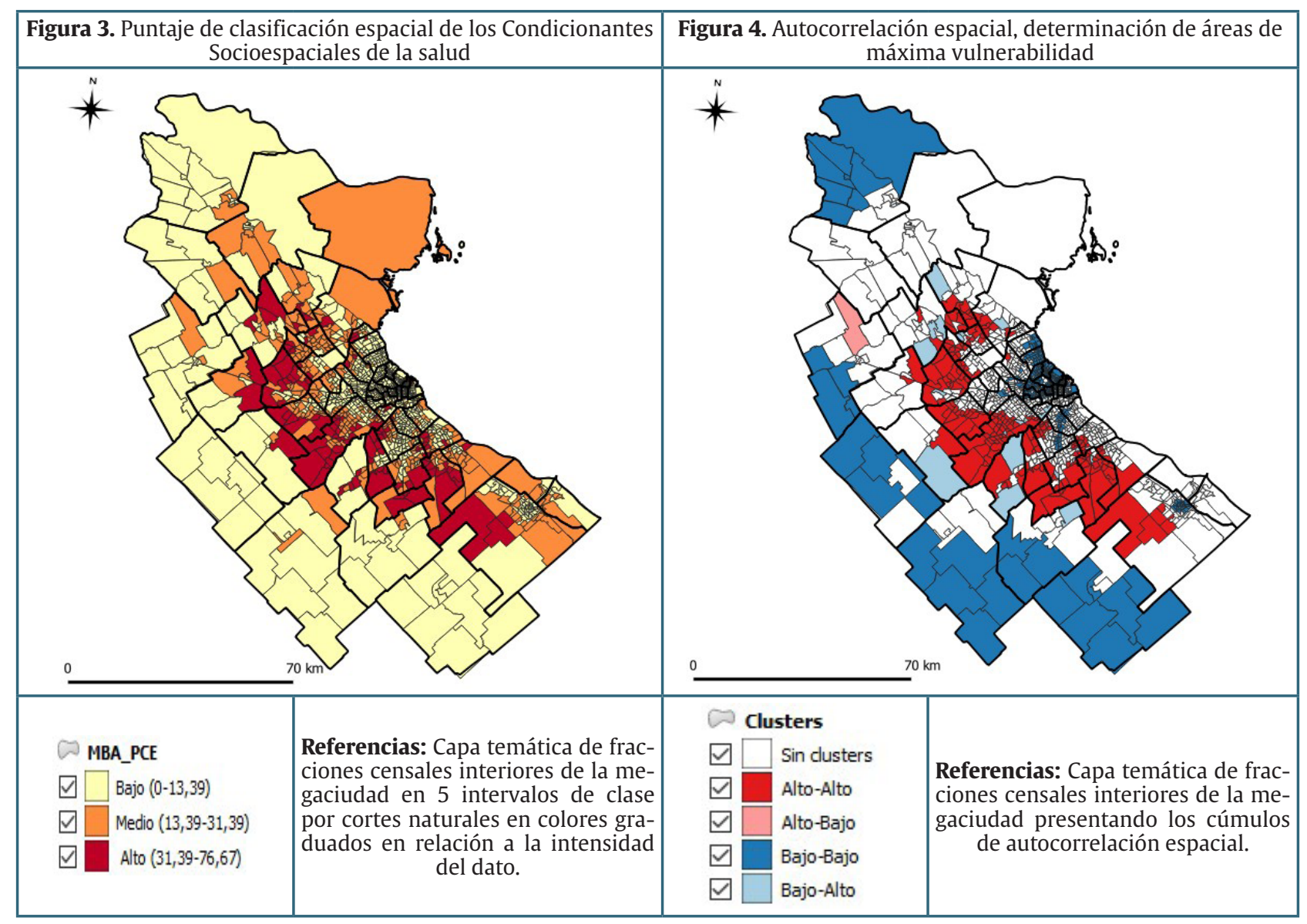

Fuente: elaboración propia.

Con base en la metodología y procedimientos aplicados, se definieron las unidades espaciales homogéneas de acuerdo a la correlación de las variables de costo y la combinación de la totalidad de dimensiones brinda como resultado el PCE, base de la autocorrelación espacial, ambos resultados finales de la aplicación.

El mapa síntesis refleja la distribución del PCE con colores más oscuros en las áreas de mayor vulnerabilidad (rojo), aquellas en las que la población está más propensa a adquirir enfermedades y con color más claro (beige) la situación inversa y con esta base fueron definidos dos clusters de autocorrelación espacial: áreas con valores altos rodeadas de valores altos (rojo) y áreas de valores bajos rodeadas de valores bajos (azul).

La Tabla 5 presenta datos básicos de la totalidad de municipios de la Megaciudad Buenos Aires y en su interior, tomando el análisis del hot-spot alto-alto, correspondiente a la situación de mayor vulnerabilidad de la población en base a los CSS se presenta la Tabla 6, dónde cada fracción censal determinada aparece con los datos sintéticos de su característica poblacional.

Tabla 5. Datos básicos de la Megaciudad Buenos Aires.

\begin{tabular}{|l|c|c|c|c|}
\hline \multicolumn{1}{|c|}{ Variable } & Total & Mínimo & Máximo & Promedio \\
\hline Población & 14.839 .026 & 25 & 33.919 & 11.371 \\
\hline Varones & 7.144 .729 & 17 & 16.627 & 5.474 \\
\hline Mujeres & 7.694 .297 & 4 & 17.382 & 5.896 \\
\hline $0-14$ & 3.497 .202 & 1 & 11.326 & 2.680 \\
\hline 15-64 & 9.697 .844 & 20 & 22.369 & 7.431 \\
\hline 65 y más & 1.643 .980 & 0 & 2.760 & 1.260 \\
\hline Población NBI & 1.703 .297 & 0 & 9.473 & 1.305 \\
\hline Hogares NBI & 405.588 & 0 & 2.264 & 311 \\
\hline
\end{tabular}

Fuente: elaboración propia. 
Tabla 6. Datos de las áreas de máxima vulnerabilidad.

\begin{tabular}{|l|c|c|c|c|c|}
\hline \multicolumn{1}{|c|}{ Variable } & Total & \% & Mínimo & Máximo & Promedio \\
\hline Población & 4.077 .710 & 27,48 & 6353 & 33.919 & 19.326 \\
\hline Varones & 2.023 .336 & 28,32 & 3.244 & 16.627 & 9.589 \\
\hline Mujeres & 2.054 .374 & 26,70 & 4 & 17.382 & 5.896 \\
\hline $0-14$ & 1.222 .388 & 34,95 & 2.328 & 11.326 & 5.793 \\
\hline $15-64$ & 2.617 .062 & 26,99 & 3.793 & 22.369 & 12.403 \\
\hline 65 y más & 238.260 & 14,49 & 144 & 2.469 & 1.129 \\
\hline Población NBI & 783.993 & 46,03 & 1.058 & 9.473 & 3.716 \\
\hline Hogares NBI & 170.134 & 41,95 & 251 & 2.264 & 806 \\
\hline
\end{tabular}

Fuente: elaboración propia.

Los datos globales de mayor vulnerabilidad indican desde un punto de vista espacial que las fracciones censales que presentan mayor vulnerabilidad forman un semi-círculo que se apoya principalmente en el segundo anillo de la megaciudad considerando a la CABA como municipio central y a sus municipios contiguos como primer anillo.

Las unidades espaciales con población en situación de máxima vulnerabilidad concentra el 27,48\% de la población con un $34,95 \%$ de niños y $14,49 \%$ de ancianos. Allí se ubica el $46,03 \%$ de la población con NBI en $1.880 \mathrm{~km} 2$, es decir, en el 13,25\% de la superficie del área de estudio.

Es importante señalar que la mayor parte de los problemas de salud están vinculados de una u otra manera a las condiciones socioeconómicas de la población que los padece, por lo que ninguna sociedad se encuentra exenta de ser blanco de alguna enfermedad en específico, por ejemplo, al tener malas condiciones socioeconómicas pueden padecer enfermedades tradicionales como lo son las gastrointestinales o respiratorias, mientas que las unidades con mejores condiciones pueden ser afectadas por enfermedades relacionadas con estilos de vida.

\section{Consideraciones finales}

A lo largo del desarrollo del trabajo se ha realizado la aplicación del análisis espacial cuantitativo en el estudio de los CSS, mostrando su importancia para definir las áreas prioritarias de atención y las características poblacionales incluidas en ellas.

De igual modo, la aplicación de la metodología de análisis multivariado y la obtención de los PCE combinado con el análisis de autocorrelación espacial muestra un camino metodológico eficiente para diagnosticar las condiciones sociespaciales de la población en apoyo a la toma de decisiones en el ámbito de las políticas públicas.

Los seis mapas de las dimensiones consideradas, el mapa síntesis y el mapa de cúmulos de autocorrelación espacial constituyen una herramienta de gran utilidad como aporte de la Geografía Aplicada. Sus resultados brindan indicaciones claras desde un punto de vista espacial y cuantitativo acerca de las áreas prioritarias de intervención. Los mapas presentados determinan estas características en la Megaciudad Buenos Aires.

En este sentido, la importancia de comprender espacialmente los DSS desde el trabajo de aplicación concreto permite definir estrategias que ayuden a equilibrarlos en la búsqueda de equidad espacial para la población. Los resultados son estructurales y actuar sobre ellos permitirá disminuir la vulnerabilidad sanitaria en el área de estudio. Los resultados son concretos y el ámbito de la gestión política tiene la posibilidad de incorporarlos como herramienta para la planificación como insumo de una Geografía aplicable puesta a disposición del proceso de toma de decisiones.

\section{Referencias Bibliográficas}

Alvarez Castaño, L.E. (2009). Los determinantes sociales de la salud: más allá de los factores de riesgo. Revista Gerencia y Políticas de Salud, 8(17), 69-79. Recuperado de: https://revistas.javeriana.edu.co/ index.php/gerepolsal/issue/view/307

Anselin, L. (1995). Local Indicators of Spatial Association - LISA. Geographical Analysis, 27, 93-115.

Anselin, L. 2003. An introducción to spatial autocorrelation analysis with GeoDa. Paper of the Spatial 
analysis Laboratory, Urbana-Champaign: University of Illinois.

Anthamattem, P. \& Hazen, H. (2011). An Introduction of Geography of Health. London: Blackwell.

Barcellos, Ch. \& Buzai, G.D. (2006). La dimensión espacial de las desigualdades sociales en salud: aspectos de su evolución conceptual y metodológica. Anuario de la División Geografía 2005-2006, Luján: Universidad Nacional de Luján. Luján, pp. 275-292. Recuperado de: https://adgunlu.wixsite.com/ anuario-geografia/copia-de-anuario-6-2007-2008

Barcellos, Ch., Buzai, G.D. \& Santana, P. (2018). Geografía de la Salud: bases y actualidad. (número monográfico sobre Geografía y Salud), Salud Colectiva, 14(1), 1-4. Recuperado de: https://www. scielosp.org/pdf/scol/2018.v14n1/1-4/es

Buzai, G.D. (2009). Sistemas de Información Geográfica en Geografía de la Salud. En: Pickenhayn, J.A. (Comp.) Salud y enfermedad en Geografía, Buenos Aires: Lugar Editorial, pp. 111-134.

Buzai, G.D. (2010). Análisis Espacial con Sistemas de Información Geográfica: sus cinco conceptos fundamentales. En: Buzai, G. (Ed.) Geografía y Sistemas de Información Geográfica. Aspectos conceptos y aplicaciones, Luján: Universidad Nacional de Luján, pp. 163-193.

Buzai, G.D. (2014). Mapas Sociales Urbanos. Buenos Aires: Lugar Editorial.

Buzai, G.D. (2015). Análisis Espacial en Geografía de la Salud. Buenos Aires: Lugar Editorial.

Buzai, G.D. (2020). Megaciudades de América Latina. Conceptos, modelos y Geografía de los procesos de estructuración urbana, Anuario de la División Geografia, 14:1-27. Recuperado de: https://adgunlu. wixsite.com/anuario-geografia/copia-de-anuario-13-2019

Buzai, G.D. \& Baxendale, C.A. (2011). Análisis Socioespacial con Sistemas de Información Geográfica, Tomo 1, Buenos Aires: Lugar Editorial.

Buzai, G.D. \& Baxendale, C.A. (2012). Análisis Socioespacial con Sistemas de Información Geográfica, Tomo 2, Buenos Aires: Lugar Editorial.

Buzai, G.D. \& Baxendale, C.A. (2013). Aportes del Análisis Geográfico con Sistemas de Información Geográfica como herramienta teórica, metodológica y tecnológica para la práctica del ordenamiento territorial, Persona y Sociedad, 27(2), 113-141. Recuperado de: https://personaysociedad.uahurtado.cl/index. $\mathrm{php} / \mathrm{ps} /$ article/view/43

Buzai, G.D. \& Montes Galbán, E. (2020). Megaciudad Buenos Aires: Cartografía de su última expansión y conurbación mediante el procesamiento digital de imágenes satelitales nocturnas, Revista Cartográfica, 100, 215-238. doi: 10.35424/rcarto.i100.667

Buzai, G.D. \& Santana Juárez, M.V. (2018). Condicionantes socioespaciales de la salud (CSS): bases y alcance conceptual, Anuario de la División Geografía, 12, 170-184. Recuperado de: https://adgunlu.wixsite. com/anuario-geografia/anuario-2018

Castro, J. (2011). Promoción de la salud. En: Gonzales, R.; Castro, J. \& Moreno, L. Promoción de la salud en el ciclo de vida. Mexico DF: MacGraw-Hill Interamericana.

Celemín, J.P. (2009). Autocorrelación esapcial e indicadores locales de asociación espacial. Importancia, estructura y aplicación, Revista Universitaria de Geografía, 18, 11-31. Recuperado de: http:// bibliotecadigital.uns.edu.ar/scielo.php?script=sci_arttext\&pid=S1852-42652009001100002\&lng= pt\&nrm=iso

Cordero, R. \& Murayama, C. (Eds.) (2013). Los determinantes sociales de la salud en México. Ciudad de México: Fondo de Cultura Económica.

Cromley, E.K. \& McLafferty, S.L. (2011). GIS and Public Health, New York: The Guilford Press.

Curto, S.I. (2003). Mapas en Geografía Médica, Cuaderno Prudentino de Geografia, 25, 111-129.

DPOUyT(2007) Lineamientos Estratégicos para la Región Metropolitana de Buenos Aires. La Plata: Subsecretaría de Urbanismo y Vivienda, Ministerio de Infraestructura, Vivienda y Servicios Públicos, Gobierno de la Provincia de Buenos Aires. Recuperado de: https://www.mininterior.gov.ar/planificacion/pdf/ planes-reg/Lineamientos-Estrategicos-para-la-Region-Metropolitana-de-Buenos-Aires.pdf

Emch, M.; Root, E.D. \& Carrel, M. (2017). Health and Medical Geography. New York: Guilford Press.

Fuenzalida Díaz, M.; Cobs Muñoz, V. \& Guerrero Espinosa, R. (2013). El territorio como unidad de análisis en estudios sobre las determinantes sociales de la salud, Argos, 30(59), 87-106. Recuperado de: http://ve.scielo.org/pdf/ag/v30n59/art06.pdf 
Gámir Orueta, A.; Ruiz Pérez, M. \& Seguí Pons, J.M. (1995). Prácticas de Análisis Espacial. Barcelona: Oikostau.

Garrocho, C. (1995). Análisis socioespacial de los servicios de salud, Zinacantepec: El Colegio Mexiquense.

Gatrell, A.C. \& Elliott, S.J. (2015). Geographies of Health. An Introduction. Abingdon: Routledge.

Howe, G.M. (1985) La Geografía Médica. En: Brown, E.H. (Ed.) Geografía: pasado y futuro. México DF: Fondo de Cultura Económica, 392-405.

INDEC (2003). ¿Qué es el Gran Buenos Aires?, Buenos Aires: Instituto Nacional de Estadística y Censos. Recuperado de: https://www.indec.gob.ar/dbindec/folleto_gba.pdf

INDEC (2013). Base de datos REDATAM. Base usuarios 2010. Buenos Aires: Instituto Nacional de Estadística y Censos. Recuperado de: https://www.indec.gob.ar/indec/web/Nivel4-Tema-2-41-135

Iñiguez, L. \& Barcellos, Ch. (2014). La cartografía en salud pública: viejos problemas y nuevas oportunidades. Revista do Departamento de Geografía, 390-412. doi:10.11606/rdg.v0i0.539

Kanaroglu, P.; Delmelle, E. \& Paéz, A. (2015). Spatial Analysis in Health Geography. Abingdon: Routledge.

Koch, T. (2014). Disease Maps. Chicago: The Chicago University Press.

May, J. (1950). Medical Geography: Its methods and objectives, The Geographical Review, 50, 10-41.

Mejía, L.M. (2013). Los determinantes sociales de la salud: base teórica de la salud pública. Revista de la Facultad de Salud Pública, 31, 28-36. Recuperado de: https://revistas.udea.edu.co/index.php/fnsp/ article/view/13423

Montes Galbán, E. (2020). Expansión y densificación urbana del Gran Buenos Aires (2012-2019) a partir de imágenes satelitales nocturnas. Revista Geográfica Digital. 17(33), 2-16. doi:10.30972/geo.17334097

Olivera, A. (1993). Geografía de la Salud. Madrid: Síntesis.

OMS 2010. Subsanar las desigualdades en una generación. Alcanzar la equidad sanitaria actuando sobre los determinantes sociales de la salud. Organización Mundial de la Salud. Informe final de la Comisión de los Determinantes Sociales de la Salud. Recuperado de: https://apps.who.int/iris/ handle/10665/69830

Piaget, J. (2007). La representación del mundo en el niño, Madrid: Morata.

Pickenhayn, J.A. (Comp) (2009). Salud y enfermedad en Geografía. Buenos Aires: Lugar Editorial.

Ramírez, M.L. (2005a). Las Tecnologías de la Información Geográfica aplicadas a la Planificación Territorial Sanitaria. Serie Geográfica, 12, 57-82. Recuperado de: https://ebuah.uah.es/dspace/ handle/10017/1181

Ramírez, M.L. (2005b). La moderna Geografía de la Salud y las tecnologías de la información geográfica, Investigaciones y ensayos geográficos, 4(4), 53-64. Recuperado de: https://revistaunaf.wixsite.com/ georevistafermoza/student-life

Santana, P. (2014). Introducción a la Geografía de la Salud: territorio, salud y bienestar. Toluca: Universidad Autónoma del Estado de México.

Santos Padrón, H. (2011). Los determinantes sociales, las desigualdades en salud y las políticas, como temas de investigación, Revista Cubana de Salud Pública, 37(2), 136-144.

Santana Juárez, M.V. \& Galindo Mendoza, M.G. (Eds) (2014). Geografía de la Salud sin fronteras, desde Iberoamérica. Toluca: Universidad Autónoma del Estado de México. Recuperado de: http:// ri.uaemex.mx/handle/20.500.11799/58186

Seguinot Barbosa, J. (2014). Aplicación de las geo-tecnologías en Geografía de la Salud. En: Santana Juárez, M.V. \& Galindo Mendoza, M.G. (Eds) Geografia de la Salud sin fronteras, desde Iberoamérica. Toluca: Universidad Autónoma del Estado de México, pp. 98-108. Recuperado de: http://ri.uaemex.mx/ handle/20.500.11799/58186

Snow, J. (1855). On the mode of communication of cholera. 2nd. Edition, London: John Churchill.

Sorre, M. (1952). Les fondements de la géographie humaine, Paris: Armand Colin (3 tomes).

Villerías Alarcón, I. (2017). Análisis espacial cuantitativo de los determinantes sociales de la salud en México, Geografia y Sistemas de Información Geográfica, 9(9), 216-232. Recuperado de: https:// revistageosig.wixsite.com/geosig/geosig-9-2017 